\title{
La probabilidad de contagio y deceso por COVID-19 en pacientes indígenas y no indígenas iniciando la pandemia en México
}

Jorge Enrique Horbath Corredor *

Fecha de recepción: 1 de abril de 2021

Fecha de aprobación: 2 de junio de 2021

Resumen: El objetivo de esta investigación es identificar factores de contagios y decesos por COVID-19 entre pacientes indígenas de México, para mostrar su mayor fragilidad, en contraste con pacientes no indígenas al inicio de la pandemia. Se utiliza la base de datos del 22 de mayo de 2020 de la Subsecretaría de Epidemiología de la Secretaría de Salud de México, con variables sociodemográficas, territoriales, de padecimientos, entre otras, y se elaboran modelos logísticos binarios de probabilidad de contagio y deceso. Los resultados muestran mayor riesgo de contagio y de deceso entre pacientes indígenas, con determinantes similares frente a pacientes no indígenas, pero con diferencias asociadas a los actuales lugares de residencia de la población indígena, vinculadas a ciudades intermedias y grandes ciudades, a donde migran de sus lugares de origen para trabajar principalmente en la informalidad del comercio ambulante y sin protección social, en las calles de las urbes mexicanas.

Palabras clave: Covid -19, pandemia, decesos, población indígena, demografía, modelización.

Clasificación JEL: C02, I10, J10, J15, Y10.

Cómo citar:

Horbath Corredor, J. E. (2021). La probabilidad de contagio y deceso por COVID-19 en pacientes indígenas y no indígenas iniciando la pandemia en México. Apuntes Del Cenes,40(72). Págs.205 - 232. https://doi.org/10.19053/01203053.v40. n72.2021.12647

* Doctor en Ciencias Políticas y Sociales, con Maestría en Estudios de Población y Licenciatura en Economía. Investigador titular C, Departamento de Sociedad y Cultura, El Colegio de la Frontera Sur (ECOSUR), Unidad Chetumal. jhorbath@ecosur.mx iDhttp://orcid. org/0000-0002-7849-2207 


\title{
Probability of Contagion and Mortality by COVID-19 in Indigenous and Non-indigenous Patients Initiating the Pandemic in Mexico
}

\begin{abstract}
The objective of this research is to identify contagion and mortality factors by COVID-19 among indigenous patients in Mexico, showing their greater fragility in contrast to non-indigenous patients at the beginning of the pandemic. Database of May 22, 2020, of the Undersecretariat of Epidemiology of the Ministry of Health of Mexico is used, with sociodemographic, territorial, diseases variables, among others, and binary logistic models of probability of contagion and mortality are elaborated. The results show a higher risk of contagion and mortality among indigenous patients, with similar determinants compared to non-indigenous patients, but with differences related to their current places of residence for the indigenous population, linked to intermediate cities and large cities, where they migrate from their places of origin to work mainly in the informality of street vendors and without social protection, on the streets of Mexican cities.
\end{abstract}

Keywords: Covid-19, pandemic, deaths, indigenous population, demography, modeling. 


\section{INTRODUCCIÓN}

Las pandemias que ha sufrido México en su historia muestran la fragilidad de la población indígena para enfrentarlas; además, en el seguimiento al impacto que ellas tienen en tales colectivos sociales se aprecia que estos han venido padeciendo una reiterada invisibilidad que ocasiona posteriormente la pérdida de vidas. La actual pandemia de COVID-19 ocasionada por el coronavirus SARS-CoV-2 se ha extendido por todo el planeta desde que se identificó en la provincia de Wuhan (China) en diciembre de 2019. En México fue detectada el 27 de febrero de 2020 en la Ciudad de México; sin embargo, es muy probable que el virus ya circulara desde antes de esta fecha en el país. Desde ese momento se le ha realizado seguimiento diario por parte de las autoridades mexicanas de salud y se suministra la información en bases de datos.

Se considera de especial relevancia efectuar un análisis de la primera etapa de propagación del virus en el país. Para ello se utiliza la base de datos correspondiente al 22 de mayo de 2020 como acervo de información para apreciar procesos y tendencias de la propagación del virus, a fin de poder contribuir a su comprensión, especialmente en cuanto a los efectos que tiene la pandemia entre la población indígena y su diferencia con el resto de la población, toda vez que el seguimiento institucional permite incorporar algunas características de identidad de la población indígena en los pacientes que son atendidos.

En el presente documento es muy importante destacar las diferencias de los determinantes del contagio por COVID-19 entre pacientes que hablan lengua indígena respecto a quienes no la hablan, así como también el contraste que existe en el fallecimiento de pacientes por COVID-19 según esta característica de identidad indígena.

El análisis de contagio y de decesos por COVID-19 se realiza mediante la formulación de modelos logísticos bivariados referidos a ambos fenómenos en los dos grupos de pacientes indígenas y no indígenas, estableciendo el vínculo de variables que se encuentran en la base de datos y otras de tipo territorial 
que se incluyen en la información por interpolación de la identificación de los municipios de residencia que reportan.

El documento inicia con un marco teórico de conceptos y definiciones, y con el recuento histórico sobre las pandemias y sus efectos en México, seguido del planteamiento metodológico cuantitativo y el análisis multivariado de modelos logísticos, con la descripción de las fuentes de información, la formulación matemática de modelos y variables que se utilizan. Luego se muestran los principales resultados de los modelos logísticos de la variación de probabilidades de contagio y de deceso por COVID-19, su análisis y contraste poblacional en ambos grupos de pacientes indígenas y no indígenas. Por último, se exponen las conclusiones en diferencias de los determinantes tanto de contagios y de decesos en ambos grupos de pacientes.

\section{MARCO TEÓRICO}

Las declaratorias de pandemia de influenza por parte de la Organización Mundial de la Salud (OMS) han sufrido cambios a lo largo del tiempo, desde 1918 hasta el 2009, principalmente porque en los lineamientos se han modificado las fases de intensidad. La OMS define una pandemia como una infección que puede mantener la infectividad y extenderse por una región global y contra la cual la población humana no tiene inmunidad (Doshi, 2011).
De las fases de intensidad en el contagio en una pandemia enunciadas por la OMS (WHO, 2009), es a partir de la fase 4 en que hay transmisión verificada de persona a persona de un virus reordenado de influenza animal o humano-animal capaz de causar "brotes a nivel comunitario", por lo que representa un aumento significativo en el riesgo de una pandemia. En la fase 5 hay una propagación del virus de persona a persona en al menos dos países de una región de la OMS, lo que es una fuerte señal de que una pandemia es inminente y que el tiempo para finalizar la organización, comunicación e implementación de las medidas de mitigación planificadas es corto. Mientras que la fase 6 se caracteriza por brotes a nivel comunitario en al menos otro país, lo que mostrará que se está produciendo una pandemia mundial (WHO, 2009).

La actual pandemia surgió en 2019 por un nuevo virus en Wuhan, China, y se convirtió en un brote mundial como enfermedad respiratoria, lo que hizo que la prevención y la contención fueran la prioridad contra la COVID-19, así como la observación de las características distintivas de la enfermedad, para una detección temprana que permitiera su manejo y clasificación para combatirla (Baghchechi et al., 2020). Durante la pandemia se han manejado tres definiciones de contagios que corresponden a las formas en que se propaga la pandemia. La primera de ellas es la que se considera un "caso importado", 
es decir, cuando una persona adquirió la infección en un país diferente al que fue diagnosticada; la segunda definición de contagio lo denomina por "contacto estrecho", esto es, cuando una persona se ha contagiado por otra que había visitado recientemente países donde había brotes de COVID-19, por lo cual hay una relación directa con el "caso importado" (WHO, 2009). El tercer tipo de contagio es el denominado "caso autóctono", el cual muestra los contagios que se irradian en lo local y que la OMS denomina "circulación comunitaria" o "transmisión comunitaria", que es la que ocurre cuando es imposible detectar el origen y la dirección del contagio dentro de una localidad, ciudad, región o país (WHO, 2009).

Las epidemias y pandemias pueden atravesar territorios y extenderse por países y continentes como ha sucedido con el VIH, H1N1, H5N1 y SARS (Verikios et al., 2016), trayendo problemas de salud, así como desequilibrios económicos y sociales, y alteraciones políticas (Davies, 2013). Por lo que la actual pandemia de COVID-19, como las anteriores, pone en riesgo el sistema social y económico (Drake et al., 2012), como se vivió en el 2003 con el SARS y con el Ébola en 2013 en China y en 2015 en África Occidental, situación que minó las formas de vida y los procesos educativos y estructuras productivas locales y globales por hacer frente a los brotes (Nabarro \& Wannous, 2016).
Los efectos de las pandemias en la historia de México han sido devastadoras para la población indígena (Borah \& Cook, 1974; Bartolomé de las Casas, 1977, citado por Cordero del Campillo, 2001; López-Ríos, 1993; Malvido, 2003). El virus de H1N1 que apareció en México en mayo de 2009, se propagaba por contagio entre las personas (Verikios et al., 2016). La OMS comunicó que hubo más de 182 000 contagios y casi 1800 decesos en 178 países durante cuatro meses (Rewar et al., 2015), mientras que en Estados Unidos el período anualizado de abril del 2009 al mismo mes del 2010 llevó a una estimación de entre 43 y 89 millones de contagios, con entre $195000 \mathrm{y}$ 403000 casos graves que requirieron de hospitalización y como resultado hubo más de 18000 decesos (Bhandari et al., 2013). Hasta ahora, la influenza era la pandemia de mayor incidencia en morbilidad y también en mortalidad, como constata la Organización Mundial de la Salud, pues la letalidad se encuentra entre $\operatorname{los} 250000$ y 500000 decesos por año con alta propagación (WHO, 2013), lo que ha llegado a estimarse en más de 18000 fallecimientos entre el 2009 y el 2010 (Prager et al., 2016). El virus H5N1 ha contagiado a muchas personas en Asia y Europa de manera reiterada (Fangriya, 2015), con cerca de cuatro centenares de casos y una letalidad de más del $60 \%$ en más de una docena de países entre el 2003 y el 2008 (Enemark, 2009); así mismo, el virus 
H7N9 de influenza, que surgió en China (Tanner et al., 2015), ha mostrado su capacidad pandémica con más de 1200 contagiados y una letalidad superior al $40 \%$ (WHO, 2017).

El 11 de marzo del 2009 se encontró el primer caso verificado del nuevo virus A (H1N1) en la Ciudad de México, así como también un brote de enfermedad respiratoria similar a la influenza en la comunidad de La Gloria, en Perote, estado de Veracruz (CDC, 2009). Los casos sospechosos eran 80600 , 17 meses después, mientras que los confirmados ascendieron a 17416 . Fue necesario hospitalizar a 7974 y lamentablemente fallecieron 146 personas, lo que mostró una tasa de hospitalización del $59.1 \%$ para los casos entre los 15 y los 44 años de edad, con una tasa de ventilación asistida (invasiva y/o entubación endotraqueal) del $2.8 \% \mathrm{y}$ una tasa de letalidad del $0.18 \%$, aunque la intensidad del contagio mostró no ser uniforme. Los casos elevados se dieron en los estados de Yucatán, Chiapas, Colima y Tabasco (Comisión Económica para América Latina y el Caribe [CEPAL], 2010). Pese a que la pandemia se comportaría como una influenza estacional, los datos de la Secretaría de Salud de México mostraron un registro el 9 de octubre del 2009 de 36593 casos confirmados y 248 defunciones, de un total de 153
697 casos y 3406 fallecidos en el continente americano y un contagio de 35 países (PAHO, 2009).

\section{METODOLOGÍA Y FUENTES DE INFORMACIÓN}

La investigación se centra en el método cuantitativo, que analiza la información de la pandemia de la COVID-19 que recopila el Sistema Mexicano de Salud. La información se concentra en bases de datos diarias, liberada desde el portal de seguimiento epidemiológico, con datos recopilados con fundamentación científica compuesta por unidades de análisis, variables y valores cuya estructura es una matriz de datos cambiante (Galtung 1978), al aglutinar información durante la pandemia, en que el contexto de la recolección de los datos interactúa directamente con el fenómeno (2010). Esta es la primera vez que en México se ponen a disposición los datos de la información validada a diario de una pandemia.

La Dirección Epidemiológica de la Secretaría de Salud de México elaboró instrumentos para la vigilancia epidemiológica de la pandemia de COVID-19, entre los que se destaca el formato de "Estudio epidemiológico de caso sospechoso de Enfermedad por 2019-nCoV" (Secretaría de Salud, 2020b), que se diligencia al momento de atender a cada paciente y se convierte en un registro en la base de datos del día 
en que se recibió el diagnóstico en una de las unidades del sistema de salud, para ser monitoreado en la evolución de su condición de salud. La información se puso a disposición del público para consultar el acervo histórico de bases de datos y diccionarios en la sección de Datos Abiertos del portal de la Dirección General de Epidemiología (Secretaría de Salud, 2020a).

Esta base de datos inició con el primer caso detectado por el sistema de salud de México, el 27 de febrero del 2020; se comenzó la liberación de la base de datos diaria desde el 30 de abril del 2020 y se trabajó con la base de datos liberada el 22 de mayo de 2020. La Secretaría de Salud esperaba que para esa fecha llegaría el pico máximo de contagios, aunque no fue así, pues el valor de la base de datos radica en ser una de las primeras fuentes de seguimiento que recopiló el proceso inicial de la propagación del contagio por COVID-19 en la primera etapa de la pandemia en México, con 86 días de casos acumulados desde el primer caso hasta el 22 de mayo de 2020.

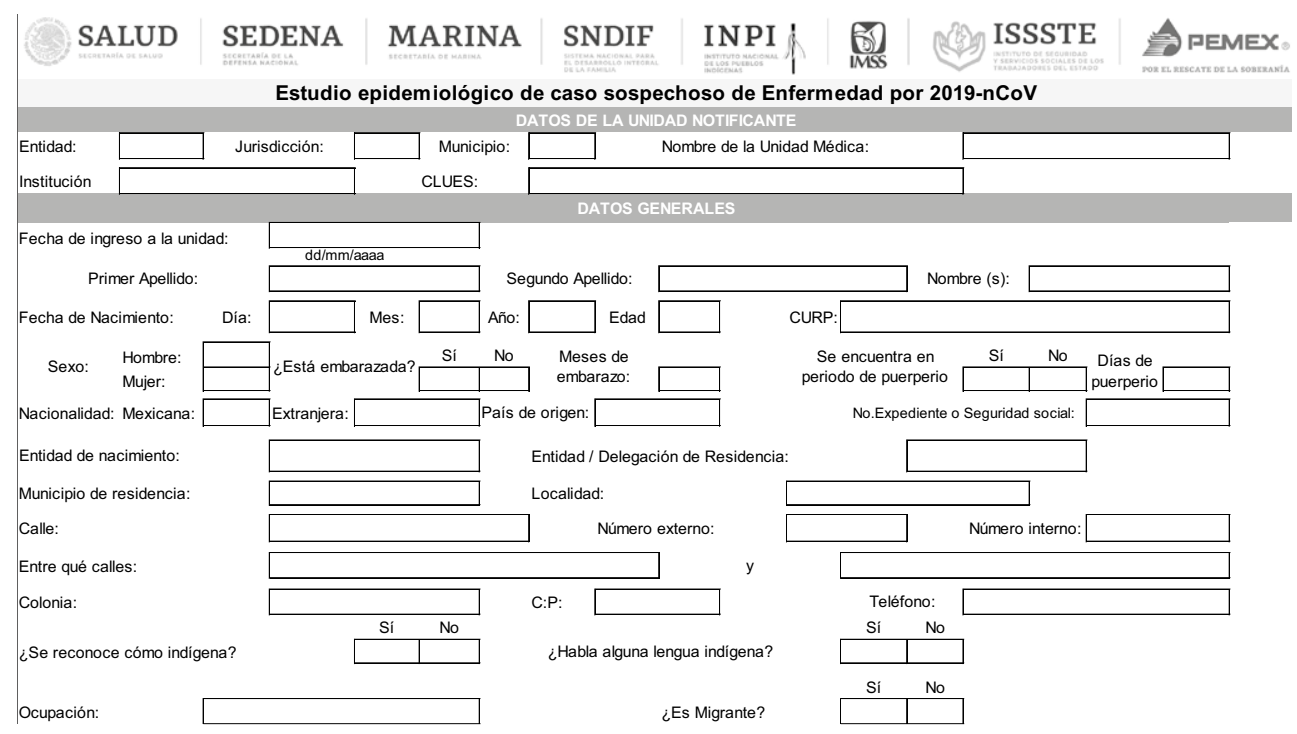

Figura 1. Formato de registro de pacientes sospechosos con COVID-19, módulos de datos generales.

Fuente: Secretaría de Salud (2020b).

Al revisar tanto la base de datos como el formato de registro de casos, se aprecia la diferencia en la información que se libera al público; primero pasa por los datos de la unidad de salud notificante, que sí son incluidos, así como algunos datos generales del paciente, entre los que no se suministran aquellos especí- 
ficos de identificación como el nombre, la CURP ni la dirección del lugar de residencia, teléfono, entre otros, que son los que se mantienen reservados en atención a la confidencialidad. El formato sí recoge algunas características sobre identidad étnica, como son las preguntas " $i S e$ reconoce como indígena? (sí, no)" y "¿Habla lengua indígena? (sí, no)" (ver Figura 1), de las cuales en la base de datos solamente se libera la segunda sobre lengua indígena como único atributo que se vincula en cada registro.

Tal situación reduce sustancialmente la identificación de la población indígena como pacientes atendidos por sus características de autorreconocimiento y queda solamente la característica histórica "hablar lengua indígena" que se ha utilizado en las instituciones del Gobierno mexicano. Este control de información fue subsanado por la Secretaría de Salud el 28 de octubre del 2020, cuando se liberó la primera pregunta de reconocimiento como indígena y empezó a figurar con la segunda pregunta de habla lengua indígena, pero hasta esa fecha las bases de datos diarias tienen ese limitante.

Con la base de datos se crean variables de identificación de pacientes por características poblacionales de identificación étnica, si son o no indígenas según su condición de habla lengua indígena, lo mismo que las características individuales como sexo y edad. La experiencia laboral del paciente se toma como el cuadrado de la edad, artificio clásico en los estudios estadísticos para la fuerza de trabajo ante la falta de información sobre ocupación. También se precisó sobre la atención que los pacientes recibieron, lo que generó una variable de tres categorías: ambulatoria, hospitalizado sin intubación y hospitalizado con intubación. Se manejaron dos bloques de variables que operaban para todos los casos registrados en la base de datos y otro juego que solamente aplicaba para casos con diagnóstico verificado con COVID-19.

Se crearon variables dicotómicas para el caso de las enfermedades como neumonía y el listado de comorbilidad; igual se hizo con el diagnóstico de COVID-19, en el que se enfatizó la comprobación de la prueba, el reporte de deceso del paciente y las condiciones de ser migrante y de ser mexicano. Se sintetizaron variables de identificación de municipios con sus claves, tanto del reporte en el diagnóstico al momento de la atención como también en el caso de la indicación del lugar de residencia.

Posteriormente, se procedió a trabajar con variables de tiempo referidas a las fechas de ingreso a la unidad de atención, así como a la fecha de inicio de los síntomas y su diferencia en días entre sí y, a su vez, respecto a la fecha de la base de datos del 22 de mayo de 2020. Igualmente se procedió con la fecha de defunción cuando el registro indicaba que el paciente había fallecido por COVID-19; se creó un juego de seis 
variables de tiempo referidas al paciente y su proceso desde los síntomas, ingreso a la unidad para su diagnóstico y comprobación de contagio con su hospitalización hasta su deceso, si se presentaba el infortunado caso del fallecimiento.

Por último, se tomaron las variables de identificación de lugar de residencia como mecanismo de enlace de características regionales, vinculadas con las tipologías de municipios de los cinco grados de marginación (Consejo Nacional de Población [CONAPO], 2016), que se calculan con la información de los censos de población y las encuestas intercensales. En este caso se utilizó la información elaborada con base en la Encuesta Intercensal de 2015 , se creó una variable de cinco categorías, que posteriormente se dicotomizaría para mayor precisión, a través de cinco variables, una por cada categoría de municipios por grado de marginación (muy alta, alta, media, baja y muy baja). La misma variable de lugar de residencia por municipios permitió incorporar la tipología de municipios indígenas del mismo año 2015 con seis categorías basadas en la clasificación de la Comisión Nacional para el Desarrollo de los Pueblos Indígenas (CDI, 2016) de México y que es retomada actualmente por el Instituto Nacional de los Pueblos Indígenas (INPI) de México, así: 1) con $70 \%$ y más es población indígena, 2) con entre 40 y $70 \%$ es población indígena, 3) con cinco mil y más habitantes indígenas, 4) con menos de cinco mil habitantes indígenas, 5) con población indígena dispersa, 6) sin población indígena. Y se crearon seis variables dicotómicas para cada categoría. Se adicionó la variable sobre presencia indígena igualmente para el 2015 con cuatro categorías referidas a municipio sin población indígena, con población indígena dispersa, con presencia indígena, y municipio indígena, que permitieron crear cuatro variables dicotómicas alusivas a cada categoría. Finalmente se agregó la variable tamaño de población municipal (INEGI, 2018) del municipio donde los pacientes referían que residían, como una variable de concentración humana y de desarrollo urbano.

Se plantean modelos logísticos binomiales para los dos grupos de pacientes: aquellos que hablan lengua indígena y quienes no hablan lengua indígena, y se contrastan sus resultados para establecer diferencias. En el caso de los modelos logísticos binarios, estos se vinculan a investigaciones que analizan los factores que registran incidencia en la mortalidad de las poblaciones mediante esta técnica para modelos de riesgo discreto (Guilkey \& Rindfuss, 1987; Zhu \& Li, 2014), usando variables demográficas como la edad, el sexo, el lugar de residencia, el diagnóstico patológico de enfermedades y la temporalidad del proceso de contagio e ingreso a las unidades de cuidados intensivos y su tipo de atención. Estas variables determinantes buscan explicar los cambios en la razón de momios 
(odds ratio, OR) que es la razón de dos odds o razones de probabilidad de ocurrencia de un evento frente a la probabilidad de que el evento no ocurra (Kleinbaum \& Klein, 2010), que en el presente estudio es el contraste de los eventos de contagiarse respecto a no contagiarse de COVID-19 y fallecer o no fallecer por COVID-19 para modelos logísticos binomiales.

\section{ANÁLISIS MULTIVARIADO DEL CONTAGIO / DECESO POR CO- VID-19 Y SUS DETERMINANTES}

A continuación, se realizará un análisis mutivariado para condensar en un modelo los resultados hasta el momento obtenidos. Nos concentraremos en identificar los posibles determinantes sociodemográficos del contagio por COVID-19 hasta el 22 de mayo de 2020.

\section{Diferencias de probabilidades de "contagiarse de COVID-19" y de "fallecer por COVID-19"}

Para establecer la probabilidad de que ocurra el evento tal que los individuos que fueron atendidos en el sistema mexicano de salud después de aplicarles la prueba de COVID-19 hayan salido positivos o negativos, así como también la condición de haberse contagiado de COVID-19 y fallecer, en contraste con no haber fallecido, respecto a sus carac- terísticas sociodemográficas propias y a algunas de las características de los espacios de asociación, para lo cual se empleó el análisis de regresión logística bivariada (RLB).

De esta manera, la formulación del modelo logístico bivariado que relaciona la razón entre las probabilidades de ocurrencia y no ocurrencia del evento "Haberse contagiado de COVID19" respecto a "No haberse contagiado de COVID-19" para el primer modelo y también la razón de probabilidades del evento "Haber fallecido por COVID19 " respecto a "No haber fallecido por COVID-19" con la condición de haberse contagiado por COVID-19 para el segundo modelo. En ambos modelos el planteamiento de razón de probabilidades viene dado por la siguiente expresión:

$$
\log \left(\frac{\Pi_{\omega}}{\Pi_{\phi}}\right)=B_{0}+B_{1} X_{1}+\cdots+B_{p} X_{p} \quad[1]
$$

Donde $\Pi_{\omega}$ es la probabilidad de que ocurra el evento "Haberse contagiado de COVID-19" y lo mismo del evento "Haber fallecido por COVID-19", y el denominador del fraccionario $\Pi_{\phi}$ es la probabilidad de que no ocurra el evento, es decir, "No haberse contagiado de COVID-19" y también "No Haber fallecido por COVID-19". 


$$
\frac{\Pi_{\omega}}{\Pi_{\phi}}=e^{B_{0}+B_{1} X_{1}+\cdots+B_{p} X_{p}}=e^{B_{0}} e^{B_{1} X_{1}} \ldots e^{B_{p} X_{p}}
$$

En ella, la probabilidad de "ocurrencia del evento" se expresaría como:

$$
\Pi_{\omega}=\frac{e^{B_{0}+B_{1} X_{1}+\cdots+B_{p} X p}}{1+e^{B_{0}+B_{1} X_{1}+\cdots+B_{p} X_{p}}}=\frac{1}{1+e^{-\left(B_{0}+B_{1} X_{1}+\cdots+B_{p} X_{p}\right)}}
$$

Y la probabilidad de "no ocurrencia del evento" sería:

$$
\Pi_{\phi}=1-\Pi_{\omega}
$$

Siendo $B_{0}, B_{1, \ldots,} B_{p}$, los parámetros que se desean estimar.

Las variables categóricas y continuas tanto dependientes como las que se seleccionaron para modelar como determinantes de los eventos "Haberse contagiado de COVID-19" y "Haber fallecido por COVID-19", así como también de la modelación de los eventos "Hospitalización sin entubar" y "Hospitalización entubado" fueron las siguientes:

\section{Variables}

(dicotómicas)

dependientes:

Indicadores resúmenes de contagio y fallecimiento comprobado por COVID-19

CONTAG_COVID19: contagiados confirmados con COVID-19, 0 NO 1 SÍ
DEFUNCIN COVID19: decesos confirmados con COVID-19, 0 NO 1 SÍ

ATENCONTAG_COVID19: contagiados COVID-19 según gravedad, 0 Ambulatorio 1 Hospitalizado sin entubar 2 Hospitalizado entubado

ATENCONTAG: atención general según gravedad, 0 Ambulatorio 1 Hospitalizado sin entubar 2 Hospitalizado entubado

Variables independientes categóricas

Características del individuo:

SEXO: sexo del paciente, 0 HOMBRE 1 MUJER

NACIONALIDAD: el paciente es mexicano o extranjero, 0 Extranjera 1 Mexicana

HABLA_LENGUA_INDIG: el paciente habla lengua indígena, 0 NO 1 SÍ

NEUMONÍA: al paciente se le diagnosticó con neumonía, 0 NO 1 SÍ 
DIABETES: el paciente tiene un diagnóstico de diabetes, 0 NO 1 SÍ

EPOC: el paciente tiene un diagnóstico de EPOC, 0 NO 1 SÍ

ASMA: el paciente tiene un diagnóstico de asma, 0 NO 1 SÍ

INMUSUPR: el paciente presenta inmunosupresión, 0 NO 1 SÍ

HIPERTENSION: el paciente tiene un diagnóstico de hipertensión, 0 NO 1 SÍ

OTRA_COM: el paciente tiene diagnóstico de otras enfermedades, 0 NO 1 SÍ

CARDIOVASCULAR: el paciente tiene un diagnóstico de enfermedades cardiovasculares, 0 NO 1 SÍ

OBESIDAD: el paciente tiene diagnóstico de obesidad, 0 NO 1 SÍ

RENAL_CRONICA: el paciente tiene diagnóstico de insuficiencia renal crónica, 0 NO 1 SÍ

TABAQUISMO: el paciente tiene hábito de tabaquismo, 0 NO 1 SÍ

OTRO_CASO: el paciente tuvo contacto con algún otro caso diagnosticado con SARS CoV-2, 0 NO 1 SÍ

MIGRANTE: el paciente es una persona migrante, 0 NO 1 SÍ
Características regionales:

TIPOMUN2015_1: municipios con $70 \%$ y más es población indígena, 0 NO 1 SÍ

TIPOMUN2015_2: municipios con entre 40 y $70 \%$ es población indígena, 0 NO 1 SÍ

TIPOMUN2015_3: municipios con cinco mil y más habitantes indígenas, 0 NO 1 SÍ

TIPOMUN2015_4: municipios con menos de cinco mil habitantes indígenas, 0 NO 1 SÍ

TIPOMUN2015_5: municipios con población indígena dispersa, 0 NO 1 SÍ

TIPOMUN2015_6: municipios sin población indígena, 0 NO 1 SÍ

PRESINDMUN2015_1: municipio sin población indígena, 0 NO 1 SÍ

PRESINDMUN2015_2: municipio con población indígena dispersa, 0 NO 1 SÍ

PRESINDMUN2015_3: municipio con presencia indígena, 0 NO 1 SÍ

PRESINDMUN2015_4: municipio indígena, 0 NO 1 SÍ

GRADMARG2015_1: municipios con grado de marginación muy bajo 2015, 0 NO 1 SÍ 
GRADMARG2015_2: municipios con grado de marginación bajo 2015, 0 NO 1 SÍ

GRADMARG2015_3: municipios con grado de marginación medio 2015, 0 NO 1 SÍ

GRADMARG2015_4: municipios con grado de marginación alto 2015, 0 NO 1 SÍ

GRADMARG2015_5: municipios con grado de marginación muy alto 2015,0 NO 1 Sí

\section{Variables continuas}

EDAD: edad en años cumplidos

EDAD2: experiencia laboral del paciente como el cuadrado de la edad

TPOBTOT: población total del municipio en que se contagió, según Censo de 2010

TIEMPO_INGRESO: días de duración entre el ingreso a diagnóstico y el 22 de mayo de 2020

TIEMPO_SINTOMAS: días de duración entre los primeros síntomas y el 22 de mayo de 2020

TIEMPO INGSINT: días de duración entre los primeros síntomas y su ingreso
TIEMPO_DEFUNCION: días de duración entre su defunción y el 22 de mayo de 2020

TIEMPO_SINT_DEF: días de duración entre los primeros síntomas y su defunción

TIEMPO_ING_DEF: días de duración entre el ingreso a diagnóstico y su defunción

La base de datos contaba con un total de 210070 pacientes registrados a nivel nacional y para el análisis de regresiones se seleccionaron los casos que reportaban resultados en las pruebas de COVID-19. En las variables del bloque de contagiados COVID-19 según gravedad, fueron analizados 62527 casos, mientras que los contagiados confirmados con COVID-19 fueron 176269 casos y los decesos confirmados con COVID-19 se contabilizaron en 9471 casos. Estos totales se mantienen o se reducen en las variables independientes, principalmente si son categóricas (ver Tabla 1); en las variables como "Habla lengua indígena", y de diagnóstico de enfermedades y padecimientos como diabetes, EPOC, asma, inmunosupresión, hipertensión, otras enfermedades, enfermedades cardiovasculares, obesidad, insuficiencia renal crónica y tabaquismo, los casos disminuyen entre el 0.67 al $2.51 \%$.

El análisis que se propone contiene un contraste no solamente de grupos que recibieron resultados de la prueba de 
COVID-19, sino que se comparan dos subgrupos de pacientes de acuerdo con la identificación como indígena según la pregunta "Habla lengua indígena", para los cuales se establece un subgrupo de pacientes que hablan lengua indígena identificándolos como "Indígenas" y otro subgrupo de pacientes que no hablan lengua indígena como "No indígenas", y se elaboran modelos para cada uno de ellos. De igual manera, se destaca que en las diversas pruebas que se realizaron con respecto a las variables de características de los municipios como "Tipo de municipio por población indígena", que tenía seis categorías, al igual que "Tipo de presencia indígena en municipio según el Censo de Población de 2015", que contaba con cuatro categorías, y "Grado de marginación de los municipios en 2015", que tenía cinco categorías, algunas de dichas categorías resultaban poco representativas o no explicativas en los modelos, por lo cual se procedió a dicotomizar cada categoría para convertirla en variable. De allí que las tres variables permitieron la creación de 15 variables dicotómicas, para facilitar así el proceso de selección a partir de los criterios estadísticos de cada modelo.

Las frecuencias absolutas se pueden apreciar en la Tabla 1, donde se muestra que, de acuerdo con la atención de pacientes tanto indígenas como no indígenas, la tasa de contagio por COVID-19 comprobada con resultados de pruebas positivas es del $35.4 \%$ de los pacientes atendidos, mientras que la tasa de letalidad se ubica en el $11.1 \%$ de los pacientes que registraron positivo en la prueba de contagio. Se tiene una tasa de contagio del $39.7 \%$ para hombres y del $31 \%$ para mujeres, y una tasa de letalidad por contagio de COVID-19 del $13.2 \%$ para hombres y del $8.3 \%$ para mujeres, lo que muestra una incidencia de contagio y de letalidad mayor entre hombres que en mujeres.

Por gravedad del padecimiento al momento de la atención que recibieron los pacientes se aprecia que el $37 \%$ de los hombres requirieron hospitalización sin intubación, el $4.6 \%$ sí lo requirieron, en tanto que entre las mujeres el $28.2 \%$ necesitó de hospitalización sin intubación y el 2.7 \% sí lo requirió.

Dentro de los pacientes según condición de habla lengua indígena, se aprecia que hay una mayor tasa de contagio entre los pacientes indígenas que entre los pacientes que no hablan lengua indígena, siendo del $43 \%$ y $35.4 \%$ respectivamente, mientras que la tasa de letalidad por COVID-19 de pacientes indígenas contagiados es el doble que la registrada entre pacientes no indígenas, correspondiente al $20.4 \%$ y $11 \%$ respectivamente. Entre los pacientes no indígenas, el $38.2 \%$ fue hospitalizado sin ser entubado y el $3.7 \%$ requirió entubarse, mientras que entre los pacientes indígenas el $41.8 \%$ fue hospitalizado sin intubación y el $5.6 \%$ sí lo requirió. 


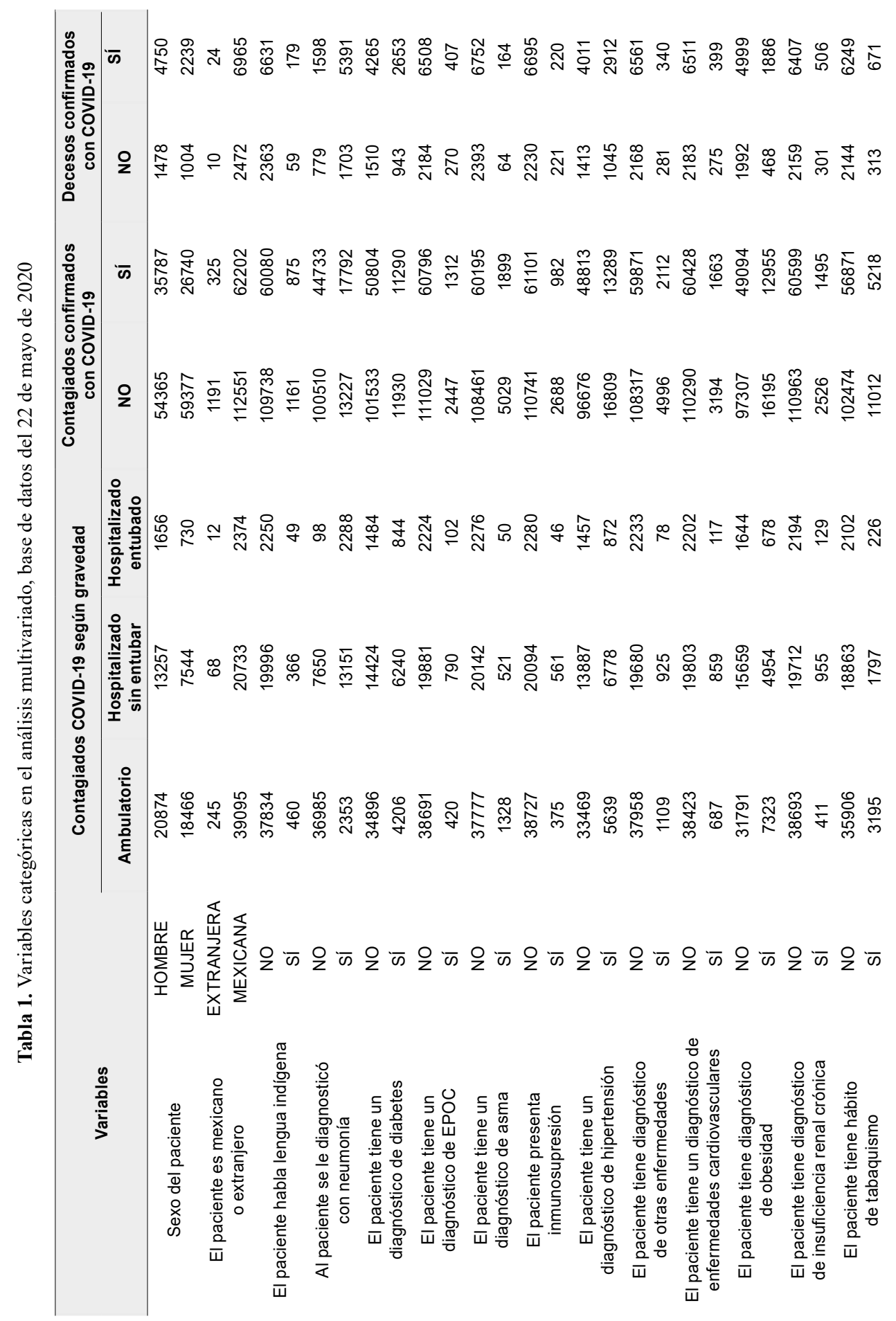




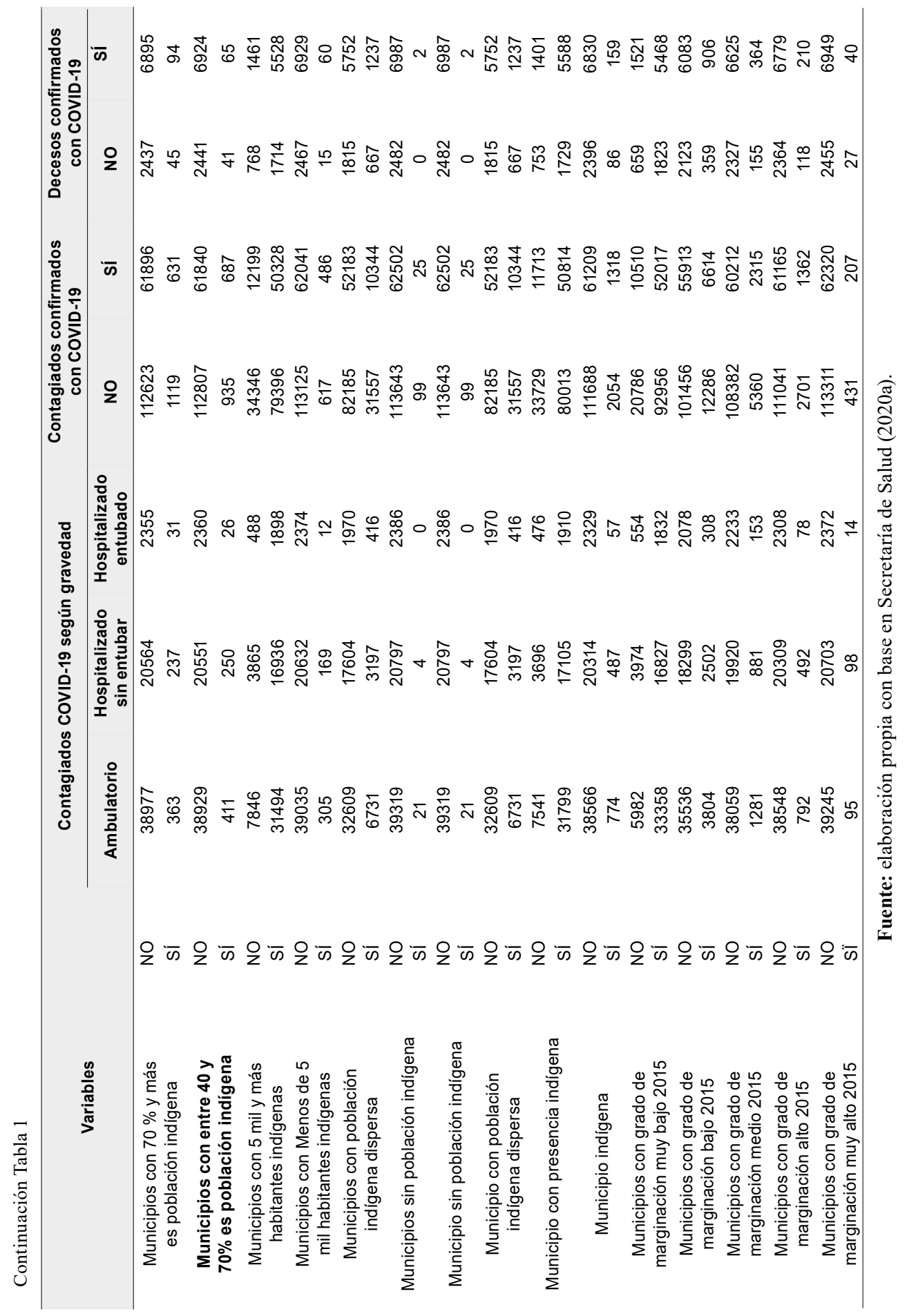


RESULTADOS DE LOS MODELOS LOGÍSTICOS BIVARIADOS DE CONTAGIO Y DE DECESO POR COVID-19

Los modelos se construyeron a partir del método de selección por pasos hacia delante, que contrasta la entrada basándose en la significación del estadístico de puntuación y contrasta la eliminación con fundamento en la probabilidad del estadístico de Wald. Las covariables categóricas son dicotómicas y fueron ingresadas con contraste polinómico con la primera categoría de referencia en cada una de ellas. El primer par de modelos que se elaboraron corresponden a regresiones logísticas binarias, cuya variable dicotómica dependiente es el contagio de COVID-19 en pacientes atendidos con resultado de la prueba, y se estableció el contraste con la variable dicotómica "Habla lengua indígena". El número de casos incluidos en el análisis para el primer modelo fue de 168 443, lo que muestra una buena verosimilitud y representatividad de la explicación de las variables respecto al fenómeno que alcanza a ser del $18 \%$, lo cual es significativo respecto al momento inicial de la pandemia, correspondiente a menos de los primeros cinco meses.
En el segundo modelo, el número de casos incluidos en el análisis fue de 2017 pacientes y ello se explica porque, a diferencia del primer modelo donde se seleccionaron los pacientes que refirieron no hablar lengua indígena, en el segundo modelo sí se realiza la selección de pacientes quienes indicaron hablar lengua indígena. Los resultados mostraron una parsimonia de los dos modelos, dado que se incluyeron solamente diez variables de cuarenta variables analizadas, todas ellas con niveles de significancia del $99 \%$ y con intervalos de confianza del $95 \%$ (Tabla 2).

En el primer modelo se realizaron 24 pasos, pero dado que el cambio adicional y su aporte marginal en los $\mathrm{R}$ cuadrados a partir del décimo paso no fue significativo, se procedió a seleccionar el resultado de ese paso, donde las variables incluidas en la ecuación fueron Sexo, Neumonía, Edad, Inmunosupresión, Tabaquismo, Tipo de municipio 5, Edad al cuadrado, Tiempo de ingreso respecto al 22 de mayo de 2020 y Tiempo de ingreso respecto al momento en que se presentaron los primeros síntomas; todas las variables resultaron significativas en un $99 \%$ en ambos modelos. 
Tabla 2. Modelos logísticos binarios de probabilidad de contagio de COVID-19 en pacientes atendidos con resultado de la prueba, contrastando que hablan y no hablan lengua indígena, 22 de mayo de 2020.

\begin{tabular}{|c|c|c|c|c|c|c|c|c|}
\hline \multirow{2}{*}{$\begin{array}{c}\text { Variables en la } \\
\text { ecuación }\end{array}$} & \multirow{2}{*}{ B } & \multirow{2}{*}{ E.T. } & \multirow{2}{*}{ Wald } & \multirow{2}{*}{ GI. } & \multirow{2}{*}{ Sig. } & \multirow{2}{*}{$\operatorname{Exp}(B)$} & \multicolumn{2}{|c|}{ I.C. $95.0 \%$ para EXP(B) } \\
\hline & & & & & & & Inferior & Superior \\
\hline \multicolumn{9}{|c|}{ EL PACIENTE NO HABLA LENGUA INDIGENA } \\
\hline Constante & -1.635 & .035 & 2221.887 & 1 & 0.000 & .195 & & \\
\hline SEXO & -.329 & .011 & 882.739 & 1 & .000 & .719 & .704 & .735 \\
\hline NEUMONÍA & 1.026 & .015 & 4776.228 & 1 & 0.000 & 2.790 & 2.710 & 2.872 \\
\hline EDAD & .054 & .001 & 1421.918 & 1 & 0.000 & 1.056 & 1.053 & 1.059 \\
\hline INMUSUPR & -.505 & .042 & 146.043 & 1 & .000 & .604 & .556 & .655 \\
\hline OBESIDAD & .300 & .014 & 431.611 & 1 & .000 & 1.349 & 1.312 & 1.388 \\
\hline TABAQUISMO & -.312 & .019 & 259.804 & 1 & .000 & .732 & .704 & .760 \\
\hline TIPOMUN2015_5 & -.634 & .014 & 2130.592 & 1 & 0.000 & .531 & .517 & .545 \\
\hline EDAD2 & -.0004 & .000 & 817.326 & 1 & .000 & .9996 & 1.000 & 1.000 \\
\hline TIEMPO_INGRESO & -.024 & .000 & 4891.796 & 1 & 0.000 & .976 & .976 & .977 \\
\hline TIEMPO_INGSINT & .079 & .002 & 2135.470 & 1 & 0.000 & 1.082 & 1.079 & 1.086 \\
\hline \multicolumn{3}{|c|}{ Incluidos en el análisis } & 168443 & \multicolumn{4}{|c|}{-2 log de la verisimilitud } & 194910.86 \\
\hline \multicolumn{2}{|c|}{ Casos perdidos } & & 33661 & \multicolumn{4}{|c|}{ R cuadrado de Cox y Snell } & 0.1309 \\
\hline \multicolumn{3}{|c|}{ Casos no seleccionados } & 7966 & \multicolumn{4}{|c|}{ R cuadrado de Nagelkerke } & 0.1801 \\
\hline \multicolumn{9}{|c|}{ EL PACIENTE HABLA LENGUA INDÍGENA } \\
\hline Constante & -2.119 & .336 & 39.894 & 1 & .000 & .120 & & \\
\hline SEXO & -.608 & .099 & 37.469 & 1 & .000 & .545 & .448 & .662 \\
\hline NEUMONIA & .625 & .114 & 30.095 & 1 & .000 & 1.868 & 1.494 & 2.335 \\
\hline EDAD & .089 & .013 & 43.879 & 1 & .000 & 1.093 & 1.065 & 1.122 \\
\hline EPOC & -.697 & .228 & 9.322 & 1 & .002 & .498 & .318 & .779 \\
\hline OBESIDAD & .351 & .132 & 7.050 & 1 & .008 & 1.420 & 1.096 & 1.840 \\
\hline TIPOMUN2015_5 & -.523 & .164 & 10.201 & 1 & .001 & .593 & .430 & .817 \\
\hline GRADMARG2015_5 & -.452 & .153 & 8.689 & 1 & .003 & .636 & .471 & .859 \\
\hline EDAD2 & -.001 & .000 & 31.184 & 1 & .000 & .999 & .999 & 1.000 \\
\hline TIEMPO_INGRESO & -.028 & .003 & 69.765 & 1 & .000 & .973 & .967 & .979 \\
\hline TIEMPO_INGSINT & .093 & .017 & 31.579 & 1 & .000 & 1.097 & 1.062 & 1.133 \\
\hline \multicolumn{3}{|c|}{ Incluidos en el análisis } & 2017 & \multicolumn{4}{|c|}{-2 log de la verisimilitud } & 2396.508 \\
\hline \multicolumn{3}{|c|}{ Casos perdidos } & 312 & \multicolumn{4}{|c|}{ R cuadrado de Cox y Snell } & 0.1624 \\
\hline \multicolumn{3}{|c|}{ Casos no seleccionados } & 207741 & \multicolumn{4}{|c|}{ R cuadrado de Nagelkerke } & 0.2181 \\
\hline
\end{tabular}

Fuente: elaboración propia con base en Secretaría de Salud (2020a).

En el segundo modelo se efectuaron 14 pasos y, al igual que en el primero, también se seleccionó el resultado en el décimo paso debido al bajo aporte marginal en los R cuadrados, con variables significativas dentro de la ecuación 
tales como Sexo, Neumonía, Edad, EPOC, Obesidad, Tipo de municipio 5, Grado de Marginalidad 5, Edad al cuadrado, Tiempo de ingreso respecto al 22 de mayo de 2020 y Tiempo de ingreso respecto al momento en que se presentaron los primeros síntomas, lo cual guarda semejanza con una gran parte de las variables comunes con el primer modelo.

En la interpretación de los resultados destacaremos las variables significativas que son comunes en ambos modelos, iniciando con la variable Sexo del paciente, cuyo coeficiente es negativo, lo que indica que la condición de ser mujer (la referencia es la categoría de hombre) implica que se reduce en casi un $28 \%$ el riesgo de contagio por COVID-19 para el grupo de población que no habla lengua indígena y se disminuye en un poco más del $45.5 \%$ entre quienes hablan lengua indígena. Es decir que, para el 22 de mayo de 2020, el riesgo de contagio era mayor entre los hombres que entre las mujeres, pero era muchísimo mayor para la población indígena, que en el modelo se encuentra representada por la condición de hablar lengua indígena, casi duplicando el cambio en el riesgo de la población que no habla lengua indígena.

La segunda variable que es común en ambos modelos es el diagnóstico de neumonía que, al resultar positivo, mostró un alto aporte en el riesgo de contagio por COVID-19 en ambos gru- pos de población, siendo mayor entre la población no indígena al duplicar el riesgo de contagio, mientras que entre la población indígena su efecto de incremento en el riesgo sería de casi el $86.8 \%$. Algo similar ocurre con la condición de obesidad que, al hacerse presente, incrementaba el riesgo de contagio de COVID-19, siendo mayor el aumento del riesgo entre la población indígena en un $42 \%$, respecto al incremento del riesgo en un $35 \%$ entre quienes no hablan lengua indígena. De igual manera, la edad de los pacientes mostró elevar el riesgo de contagio por COVID-19 en valores cercanos en ambos grupos poblacionales, aumentando en un $5.5 \%$ por cada año adicional entre la población no indígena y en un $9.3 \%$ entre la población indígena. En tanto que la experiencia laboral, en la que a falta de información directa se calcula una variable proxi con el cuadrado de la edad, reduce el riesgo de contagio en ambas poblaciones, pues fue mayor la reducción entre la población indígena en un $0.07 \%$ respecto al $0.04 \%$ de la población no indígena.

Otras dos variables que resultaron comunes y con igual signo de relación en ambos modelos fueron el "Tiempo de ingreso respecto a la fecha del 22 de mayo de 2020" y el "Tiempo de ingreso respecto a los primeros síntomas de la enfermedad". En el primer caso, correspondiente a los días de duración entre el ingreso a diagnóstico y el 22 de mayo de 2020, por cada día adicional el riesgo se reducía en un $2.4 \%$ entre los no indí- 
genas y en un $2.7 \%$ entre la población indígena, mientras que en la segunda variable "Días de duración entre los primeros síntomas y su ingreso", un día adicional incrementaba el riesgo de contagio por COVID-19 en un $8.2 \%$ entre quienes no hablan lengua indígena y en un $9.7 \%$ entre la población que habla lengua indígena. La última variable en común dentro de ambos modelos resultantes es la condición de que el paciente viva en un municipio con población indígena dispersa, aspecto que reduce el riesgo de contagio por COVID-19 en un $46.9 \%$ en la población no indígena y en un $40.7 \%$ entre la población indígena.

Entre las variables que resultaron disímiles en los dos modelos podemos apreciar que entre los pacientes que manifestaron no hablar lengua indígena la condición de presentar inmunosupresión reduce el riesgo de contagio en un $39.6 \%$; así como también ocurre con la condición de tabaquismo, que reduce el riesgo en un $26.8 \%$ en el mismo grupo poblacional. Mientras que entre los pacientes que hablan lengua indígena, el diagnóstico de enfermedad pulmonar obstructiva crónica (EPOC) reducía en un $50.2 \%$ el riesgo de contagio, así como también el vivir en municipios con grado de marginación muy alto hace disminuir el riesgo en un $36.3 \%$.
En cuanto al resultado de los modelos de probabilidad de defunción por COVID-19, se encontraron disimilitudes mucho más amplias tanto en el número como en el tipo de variables representativas (ver Tabla 3). Para el primer modelo con pacientes que no hablan lengua indígena fueron necesarios 19 pasos de ingreso de variables, pero se seleccionó el resultado arrojado en el paso 16, debido a que las contribuciones marginales a los $\mathrm{R}$ cuadrado eran muy bajas, y se obtuvieron 14 variables explicativas, incluyendo 8779 casos en el análisis, mientras que en el segundo modelo con pacientes que manifestaron hablar lengua indígena solamente se realizaron cinco pasos y el resultado fue mucho más parsimonioso, pues se obtuvieron cinco variables explicativas, con un número de 232 casos incluidos en el análisis. Las variables resultantes tienen una significancia del $99 \%$ en el primer modelo y entre el 99 y $97 \%$ en el segundo modelo. Del cúmulo de variables, solamente dos, la condición de neumonía y el diagnóstico de otras enfermedades, resultaron comunes en ambos modelos.

La condición de presentar neumonía en ambos grupos de pacientes eleva el riesgo de fallecer por COVID-19, con aumento de probabilidades del $49 \%$ para la población no indígena y de dos veces más para la población indígena; mientras que otras enfermedades adi- 
cionales (diferentes a las representadas en variables específicas) hacen reducir el riesgo de muerte por COVID-19 en un $38.3 \%$ para pacientes no indígenas $y$ en un $77 \%$ entre pacientes indígenas.

Siguiendo el análisis de las variables disímiles con el segundo modelo, la variable "Sexo" indica que si la persona no indígena es mujer, el riesgo de fallecer por COVID-19 disminuye en un $27.1 \%$, mientras que un año adicional en la edad de los pacientes no indígenas contagiados con COVID-19 eleva el riesgo de morir en un $10.4 \%$, y el aumento de la experiencia laboral (el cuadrado de la edad como proxi) hace disminuir en un $0.09 \%$ el riesgo de perder la vida por el contagio en pacientes no indígenas.

Cuatro condiciones de enfermedades registraron una reducción en el riesgo de fallecer por COVID-19; si se presenta inmunosupresión, el riesgo disminuye en un $48.5 \%$; si existen enfermedades cardiovasculares, se reduce la probabilidad de morir en un $34.4 \%$; así como un diagnóstico de insuficiencia renal crónica disminuye el riego de morir en un $27.1 \%$, o si hay el hábito de tabaquismo, el riesgo baja un $29 \%$ entre pacientes no indígenas. Una quinta condición de enfermedad como es la obesidad eleva el riesgo de fallecimiento por contagio de COVID-19 en un $61.6 \%$ en este grupo de pacientes.

En este mismo modelo para pacientes no indígenas las variables referidas al tiempo de síntomas, diagnóstico, internación y fallecimiento muestran que un día adicional en los días de duración entre los primeros síntomas y el 22 de mayo de 2020 eleva en un $3.4 \%$ el riesgo de fallecer, mientras que un día de más en los días de duración entre los primeros síntomas y su ingreso incrementa en un $3.6 \%$ el riesgo de morir. El riesgo de morir es menor en un $5.6 \%$ a medida que se amplía la diferencia en días desde el evento de fallecer y el 22 de mayo de 2020, debido a que las frecuencias más altas de los decesos se localizaron en fechas posteriores al 4 de mayo de 2020, muy cerca del corte de la base de datos (ver Figura 2), mientras que las frecuencias de fechas anteriores muestran números de fallecimientos marcadamente menores. Por último, si los pacientes viven en municipios con presencia indígena, esto incide en elevar en un $49.4 \%$ el riesgo de muerte por contagio de COVID-19 entre pacientes no indígenas. 
Tabla 3. Modelos logísticos binarios de probabilidad de defunción por contagio de COVID-19 en pacientes que hablan y no hablan lengua indígena atendidos con prueba positiva, 22 de mayo de 2020

\begin{tabular}{|c|c|c|c|c|c|c|c|c|}
\hline \multirow[t]{2}{*}{ Variables en la ecuación } & \multirow[t]{2}{*}{ B } & \multirow[t]{2}{*}{ E.T. } & \multirow[t]{2}{*}{ Wald } & \multirow[t]{2}{*}{ GI. } & \multirow[t]{2}{*}{ Sig. } & \multirow[t]{2}{*}{$\operatorname{Exp}(B)$} & \multicolumn{2}{|c|}{$\begin{array}{l}\text { I.C. } 95.0 \% \text { para } \\
\text { EXP(B) }\end{array}$} \\
\hline & & & & & & & Inferior & Superior \\
\hline \multicolumn{9}{|c|}{ EL PACIENTE NO HABLA LENGUA INDÍGENA } \\
\hline Constante & -1.891 & .232 & 66.554 & 1 & .000 & .151 & & \\
\hline SEXO & -.317 & .055 & 33.066 & 1 & .000 & .728 & .654 & .811 \\
\hline NEUMONÍA & .399 & .057 & 48.533 & 1 & .000 & 1.490 & 1.332 & 1.667 \\
\hline EDAD & .100 & .008 & 172.322 & 1 & .000 & 1.105 & 1.088 & 1.121 \\
\hline INMUSUPR & -.664 & .113 & 34.473 & 1 & .000 & .515 & .412 & .643 \\
\hline OTRA_COM & -.483 & .097 & 24.716 & 1 & .000 & .617 & .510 & .746 \\
\hline CARDIOVASCULAR & -.422 & .094 & 20.263 & 1 & .000 & .656 & .545 & .788 \\
\hline OBESIDAD & .480 & .065 & 54.904 & 1 & .000 & 1.617 & 1.424 & 1.836 \\
\hline RENAL_CRONICA & -.317 & .086 & 13.597 & 1 & .000 & .729 & .616 & .862 \\
\hline TABAQUISMO & -.344 & .082 & 17.571 & 1 & .000 & .709 & .604 & .833 \\
\hline PRESINDMUN2015_3 & .401 & .060 & 44.103 & 1 & .000 & 1.494 & 1.327 & 1.682 \\
\hline EDAD2 & -.001 & .000 & 165.730 & 1 & .000 & .999 & .999 & .999 \\
\hline TIEMPO_SINTOMAS & .031 & .005 & 44.222 & 1 & .000 & 1.031 & 1.022 & 1.041 \\
\hline TIEMPO_INGSINT & .036 & .009 & 15.529 & 1 & .000 & 1.036 & 1.018 & 1.055 \\
\hline TIEMPO_DEFUNCION & -.056 & .005 & 135.751 & 1 & .000 & .945 & .936 & .954 \\
\hline \multicolumn{3}{|c|}{ Incluidos en el análisis } & 8779 & \multicolumn{4}{|c|}{$-2 \log$ de la verisimilitud } & 9000.892 \\
\hline \multicolumn{2}{|c|}{ Casos perdidos } & & 193325 & \multicolumn{4}{|c|}{ R cuadrado de Cox y Snell } & 0.1181 \\
\hline \multicolumn{3}{|c|}{ Casos no seleccionados } & 7966 & \multicolumn{4}{|c|}{ R cuadrado de Nagelkerke } & 0.1727 \\
\hline \multicolumn{9}{|c|}{ EL PACIENTE HABLA LENGUA INDÍGENA } \\
\hline Constante & 1.194 & .451 & 7.012 & 1 & .008 & 3.302 & & \\
\hline NEUMONIA & 1.103 & .410 & 7.239 & 1 & .007 & 3.012 & 1.349 & 6.725 \\
\hline EPOC & -1.087 & .467 & 5.410 & 1 & .020 & .337 & .135 & .843 \\
\hline OTRA_COM & -1.469 & .605 & 5.890 & 1 & .015 & .230 & .070 & .754 \\
\hline GRADMARG2015_1 & .848 & .370 & 5.240 & 1 & .022 & 2.335 & 1.130 & 4.825 \\
\hline TIEMPO_DEFUNCION & -.044 & .014 & 10.288 & 1 & .001 & .957 & .931 & .983 \\
\hline \multicolumn{3}{|c|}{ Incluidos en el análisis } & 232 & \multicolumn{4}{|c|}{-2 log de la verisimilitud } & 228.113 \\
\hline \multicolumn{3}{|c|}{ Casos perdidos } & 2097 & \multicolumn{4}{|c|}{ R cuadrado de Cox y Snell } & 0.1318 \\
\hline \multicolumn{3}{|c|}{ Casos no seleccionados } & 207741 & \multicolumn{4}{|c|}{ R cuadrado de Nagelkerke } & 0.1953 \\
\hline
\end{tabular}

Fuente: elaboración propia con base en Secretaría de Salud (2020a). 


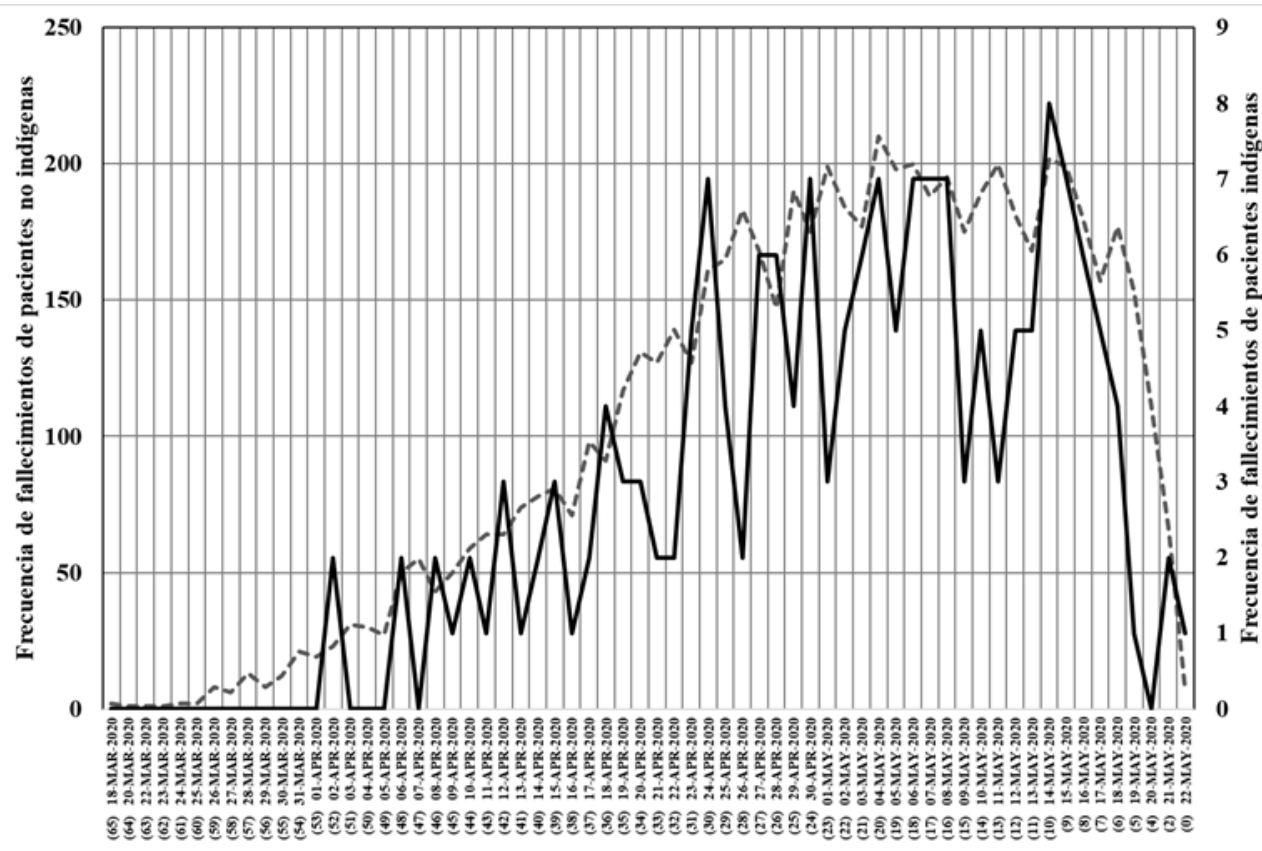

(Diferencia en dias al 22/05/2020) FECHA DE FALLECIMIENTO

--- No indigena - Indigena

Figura 2. Frecuencia de fallecimientos de pacientes contagiados por COVID-19 por fecha de ocurrencia y diferencia en días al corte del 22 de mayo de 2020.

Fuente: elaboración propia con base en Secretaría de Salud (2020).

Para el segundo modelo de pacientes que hablan lengua indígena, las tres variables restantes, que son disímiles con el primer modelo, indican que la presencia de enfermedad pulmonar obstructiva crónica (EPOC) disminuye en un $66.2 \%$ el riesgo de morir. El riesgo de fallecer es menor en $4.4 \%$ cuando aumenta la diferencia desde el deceso hasta el corte del 22 de mayo de 2020 , pues al igual que en el caso de los fallecimientos de pacientes no indígenas, las frecuencias cercanas al último día de liberación de la base de datos fueron más altas principalmente desde el 24 de abril (ver Figura 2), y las frecuencias anteriores a esa fecha fueron menores en el número de indígenas fallecidos.

\section{CONCLUSIONES}

En el recuento de las pandemias en México es la primera vez que se hace un esfuerzo por difundir día a día la información del estado de atención y contagio de la población que ha sido atendida en las unidades del sistema de salud. Su difusión a partir de las bases de datos liberadas por la Subsecretaría de Epidemiología de la Secretaría de 
Salud, vinculadas a un formato de seguimiento, también muestran que es la primera ocasión en que se aprecia la voluntad de identificar a los pacientes con características de identidad indígena. Aunque el formato contempla la tradicional característica de "habla lengua indígena", también se encuentra una segunda característica de autoadscripción como indígena, pero en la primera etapa de la pandemia las bases que se liberaron no contenían información de esa variable, por lo que se considera un subregistro institucional que invisibiliza a un gran número de pacientes indígenas que no son reconocidos como tales. Los resultados muestran que, en la primera etapa de la pandemia hasta el 22 de mayo de 2020, la mayor proporción tanto de contagios por COVID-19 como de letalidad por esta causa se registró entre pacientes indígenas mucho más que entre pacientes no indígenas; así como también que la mayor proporción de hospitalizados y el mayor requerimiento de intubación se presentaron entre pacientes que hablaban lengua indígena respecto a quienes registraron no hablarla.

En los modelos de riesgo de contagio por COVID-19 se obtuvo que este era mayor para los hombres que para las mujeres, y se elevó mucho más por la condición de ser indígena (hablar lengua indígena), lo cual casi duplica el riesgo de contagio respecto a la población no indígena (que no habla lengua indígena). Para ambos grupos de pacientes presentar neumonía elevaba el riesgo de estar contagiado por COVID19 , con un mayor aumento que duplica nuevamente el riesgo en pacientes indígenas (de más del $86 \%$ ) que en no indígenas. La obesidad también es una condición de salud que eleva el riesgo de contagio por COVID-19, cerca de diez puntos porcentuales mayor entre indígenas que entre los pacientes no indígenas. Por cada año adicional en la edad mostró un efecto positivo en el riesgo de contagio por COVID-19, al ser casi cuatro puntos porcentuales mayor en pacientes indígenas que entre los pacientes no indígenas; mientras que la experiencia laboral disminuye ese riesgo de contagio, aunque muy levemente, en menos de un punto porcentual en ambos grupos de pacientes. También, las variables de temporalidad entre los primeros síntomas de contagio y el ingreso a diagnóstico, así como la fecha de ingreso respecto a la fecha del 22 de mayo de 2020, mostraron que en el primer caso cada día adicional elevaba el riesgo de contagio entre el 8 y $9 \%$, en tanto que en el segundo caso se reducía en poco más de dos puntos porcentuales el riesgo de contagio en los dos grupos de pacientes, y vivir en un municipio con población indígena dispersa disminuye ese riesgo entre el 40 y $47 \%$ para indígenas y no indígenas respectivamente. La inmunosupresión y el tabaquismo reducen sustancialmente el riesgo de contagio entre pacientes no indígenas, mientras que la condición de enfermedad pulmonar obstructiva crónica (EPOC) y el vivir en municipios con grado de marginación muy alto 
disminuyen de manera importante el riesgo de contagio por COVID-19 en pacientes indígenas.

Los modelos de riesgo de fallecimiento por COVID-19 también mostraron que la neumonía aumenta el riesgo para ambos grupos de pacientes, con un incremento del doble para pacientes indígenas respecto a los no indígenas, y otras enfermedades diferentes a las especificadas lo reducen de una forma semejante con mayor disminución para pacientes indígenas. Ser mujer entre los pacientes no indígenas disminuye el riesgo, mientras que el aumento en la edad lo eleva, y la experiencia laboral reduce ese riesgo de manera muy leve; pero lo disminuye significativamente presentar inmunosupresión, enfermedades cardiovasculares, insuficiencia renal crónica, tabaquismo y obesidad entre pacientes no indígenas. En ellos, el aumento en días entre los primeros síntomas y el 22 de mayo de 2020, al igual que el mayor lapso entre los síntomas y el día del diagnóstico, aumentan el riesgo de fallecer por COVID-19, así como la condición de vivir en municipios con presencia indígena también eleva ese riesgo por las menores condiciones del sistema salud, y disminuye si los eventos de decesos son en fechas tempranas al corte de la base de datos, debido a que logran resistir mucho más. Para los pacientes indígenas, la presencia de enfermedad pulmonar obstructiva crónica (EPOC) reduce el riesgo de fallecer, al igual que las fechas tempranas del corte de la base de datos cuyas frecuencias de fallecimientos eran mucho menores y en las que lograban sobrevivir más tiempo; en tanto que residir en municipios con grado muy bajo de marginación, característico de medianas y grandes ciudades, eleva fuertemente el riesgo de morir por COVID-19. Lo anterior muestra la elevada exposición que tiene la población indígena migrante a los espacios urbanos en México, al realizar actividades laborales de tipo informal en las vías públicas y sin ninguna prestación social de salud.

\section{AGR ADECIMIENTOS}

Al Consejo Nacional de Ciencia y Tecnología CONACYT de México.

\section{FINANCIAMIENTO}

El presente documento contiene resultados del proyecto en curso "Exclusión, discriminación y pobreza de los indígenas urbanos en México: segunda fase de continuación de proyecto", apoyado por la Convocatoria CB-2016-01 CONACYT de México.

\section{DECLARACIÓN DE CONFLICTOS DE INTERESES}

Sin conflicto de intereses. 


\section{REFERENCIAS}

[1] Baghchechi, M., Jaipaul, N., \& Jacob, S. E. (2020). The Rise and Evolution of COVID-19. International Journal of Women's Dermatology, 6(4), 248-254. https:// doi.org/10.1016/j.ijwd.2020.06.006

[2] Bartolomé de las Casas (1977). Brevissima relación de la destruyción de las Indias, Sevilla, 1552. Facsímil.

[3] Bhandari, R., Hartley, T. A., Lindsley, W. G., Fisher, M. A., \& Palmer, J. E. (2013). Assessing Healthcare Utilization for Influenza-like Illness at an Emergency Department and a Student Health Service during the 2009-2010 H1N1 Pandemic. Infectious Diseases, 6, 15-23. https://doi.org/10.4137/IDRT.S11315

[4] Borah, W. \& Cook, S. F. (1974). Ensayos sobre historia de la población: México y el Caribe. Siglo XXI Editores.

[5] CDC. (2009). H1N1 Vaccination Recommendations. http://www.cdc.gov/h1n1flu/ vaccination/acip.htm

[6] Comisión Nacional para el Desarrollo de los Pueblos Indígenas -CDI-. (2016). Indicadores socioeconómicos de los pueblos indígenas de México, 2015. Coordinación General de Planeación y Evaluación. https://www.gob.mx/cms/ uploads/attachment/file/239921/01-presentacion-indicadores-socioeconomicos-2015.pdf

[7] Consejo Nacional de Población -CONAPO-. (2016). Índice de marginación por entidad federativa y municipio 2015. https://www.gob.mx/cms/uploads/attachment/file/159052/01_Capitulo_1.pdf.

[8] Comisión Económica para América Latina y el Caribe -CEPAL-. (2010). Evaluación preliminar del impacto en México de la influenza AH1N1. CEPAL-OPS.

[9] Cordero del Campillo, M. (2001). Las grandes epidemias en la América colonial. Revista Archivos de Zootecnia, 50(192), 597-612.

[10] Davies, S. E. (2013). National Security and Pandemics. UN Chronicle, 50(2), 20-24. https://doi.org/10.18356/0dfec716-en

[11] Doshi, P. (2011). The Elusive Definition of Pandemic Influenza. Bull World Health Organ, 89(7), 532-8. https://doi.org/10.2471/BLT.11.086173 
[12] Drake, T. L., Chalabi, Z., \& Coker, R. (2012). Cost-Effectiveness Analysis of Pandemic Influenza Preparedness: What's Missing? Bulletin of the World Health Organization, 90(12), 940-941. https://doi.org/10.2471/BLT.12.109025

[13] Enemark, C. (2009). Is Pandemic Flu a Security Threat? Survival, 51(1), 191-214. https://doi.org/10.1080/00396330902749798

[14] Fangriya, R. (2015). Pandemic Influenza Threat. World Scientific News, 11, 120-137.

[15] Galtung, J. (1978). Teorías y técnicas de la investigación social. Eudeba.

[16] Guilkey, D. \& Rindfuss, R. (1987). Logistic Regression Multivariate Life Tables: A Communicable Approach. Sociological Methods and Research 16(2), 276-300. https://doi.org/10.1177/0049124187016002004

[17] INEGI. (2018). Encuesta Intercensal 2015. Marco conceptual. Instituto Nacional de Estadística y Geografía. http://internet.contenidos.inegi.org.mx/ contenidos/Productos/prod_serv/contenidos/espanol/bvinegi/productos/nueva_ estruc/702825098742.pdf

[18] Kleinbaum, D. \& Klein, M. (2010). Logistic Regression: A Self-Learning Text. Statistics for Biology and Health. Springer. https://doi. org/10.1007/978-1-4419-1742-3

[19] López-Ríos, F. (1993). Medicina naval española en la época de los descubrimientos. Labor.

[20] Malvido, E. \& Viesca, C. (1985). La epidemia de cocoliztli de 1576. Historias, (11), 27-34. https://revistas.inah.gob.mx/index.php/historias/article/view/15223

[21] Nabarro, D., \& Wannous, C. (2016). The Links Between Public and Ecosystem Health in Light of the Recent Ebola Outbreaks and Pandemic Emergence. EcoHealth, 13(2), 227-229. https://doi.org/10.1007/s10393-016-1123-y

[22] PAHO. (2009, 9 oct.). Actualización regional. Pandemia (H1N1) 2009. https://www. paho.org/hq/dmdocuments/2009/IR_Pandemia_H1N1_2009_October9_2009.pdf

[23] Prager, F., Wei, D., \& Rose, A. (2017). Total Economic Consequences of an Influenza Outbreak in the United States. Risk Analysis: An Official Publication of the Society for Risk Analysis, 37(1), 4-19. https://doi.org/10.1111/risa.12625 
[24] Rewar, S., Mirdha, D., \& Rewar, P. (2015). Treatment and Prevention of Pandemic H1N1 Influenza. Annals of Global Health, 81(5), 645-653. http://dx.doi. org/10.1016/j.aogh.2015.08.014

[25] Secretaría de Salud. (2020a). Datos abiertos de casos diarios por municipio sobre COVID19 México. Dirección General de Epidemiología, Secretaría de Salud de México. https:/www.gob.mx/salud/documentos/datos-abiertos-152127

[26] Secretaría de Salud. (2020b). Lineamiento estandarizado para la vigilancia epidemiológica y por laboratorio de enfermedad por 2019-nCOV. Subsecretaría de Prevención y Promoción de la Salud, Dirección General de Epidemiología. México. https://www.gob.mx/cms/uploads/attachment/file/533167/Lineamiento_2019_ nCoV_2020_02_07.pdf

[27] Tanner, W., Toth, D., \& Gundlapalli, A. V. (2015). The Pandemic Potential of Avian Influenza A (H7N9) Virus: A Review. Epidemiology and Infection, 143(16), 3359-3374. https://doi.org/10.1017/S0950268815001570.

[28] Verikios, G., Sullivan, M., Stojanovski, P., Giesecke, J. \& Woo, G. (2016), Assessing Regional Risks from Pandemic Influenza: A Scenario Analysis. The World Economy, 39(8), 1225-1255. https://doi.org/10.1111/twec.12296

[29] WHO. (2009). Pandemic Influenza Preparedness and Response: A WHO Guidance Document. World Health Organization. https://www.ncbi.nlm.nih.gov/books/ NBK143061/figure/ch4.f1/?report=objectonly\%2F

[30] WHO. (2013). Pandemic Influenza Risk Management, A WHO Guide to Inform \& Harmonize National \& International Pandemic Preparedness and Response. WHO Interim Guidance. World Health Organization. https://www.who.int/influenza/preparedness/pandemic/GIP_Pandemic InfluenzaRiskManagementInterimGuidance_Jun2013.pdf

[31] WHO. (2017). Human Infection with Avian Influenza A(H7N9) Virus-China. http:// www.who.int/csr/don/22-february-2017-ah7n9-china/en/

[32] Zhu, Z. \& Zhi, L. (2014). Logistic Regression for Insured Mortality Experience Studies. In The Living to 100 Symposium, January 8-10, Orlando, Florida. 